\title{
Clinical comparison of brachytherapy versus hypofractionated external beam radiation versus standard fractionation external beam radiation for non-melanomatous skin cancers
}

\author{
Justin M Haseltine', Matthew Parker!, A. Gabriella Wernicke, MD, MSc', Dattatreyudu Nori, MD!, Xian Wu, MPH², \\ Bhupesh Parashar, MD' \\ 'Department of Radiation Oncology, Weill Cornell Medical Center, New York-Presbyterian Hospital, ${ }^{2}$ Department of Healthcare Policy \\ and Research, Weill Cornell Medical College, New York, NY, USA
}

\begin{abstract}
Purpose: Non-melanomatous skin cancer (NMSC) is the single most common cancer in the US. Radiation therapy is an excellent treatment alternative to surgery. High-dose-rate (HDR) brachytherapy and external beam radiotherapy (EBRT) are commonly used radiation treatment modalities but little data is published comparing these modalities. We present our institution's experience and outcomes with these therapeutic options.

Material and methods: From June 2005 to March 2013, 61 patients were treated with HDR brachytherapy $(n=9)$, hypofractionated EBRT $(n=30)$, or standard fractionation EBRT $(n=22)$ for NMSC. The primary outcome measure was local control at most remote follow-up and secondary outcome measures were overall survival, cosmetic outcome, and toxicity. Univariate analysis was performed to compare outcomes between treatment modalities. Kaplan-Meier analysis and log-rank test were used to compare overall survival.

Results: Median follow-up was 30 months. The most common histologies were BCC (47\%) and SCC (44\%); mean patient age was 83.3 years. Local control was $81 \%$ and 2 -year actuarial overall survival was $89 \%$. There was no statistical difference in local control or overall survival between treatment modalities. There was no statistical difference in cosmetic outcome or toxicity between treatment modalities, although five of six "poor" cosmetic outcomes and the only grade 3 toxic events were found in the standard fractionation EBRT group.

Conclusions: All modalities investigated represent effective treatments for NMSC and have good cosmetic outcomes and acceptable toxicity profiles. The finding of higher grade toxicity and a greater portion of patients experiencing toxicity among standard fractionation therapy is counter to expectations. There was no statistical significance to the finding and it is not likely to be meaningful.
\end{abstract}

J Contemp Brachytherapy 2016; 8, 3: 191-196 DOI: $10.5114 / j \mathrm{jcb} .2016 .60598$

Key words: brachytherapy, EBRT, HDR, skin cancer.

\section{Purpose}

Non-melanomatous skin cancer (NMSC) is the single most common cancer in the US with an incidence of 2-3 million cases per year [1]. NMSC is comprised of basal cell carcinoma (BCC) and squamous cell carcinoma (SCC), and also includes Merkel cell carcinoma (MCC) and adnexal tumors [2]. While surgical excision is the standard of care, radiotherapy (RT) is an excellent alternative with high cure rates and good cosmetic outcome $[3,4,5$, $6,7,8,9,10,11,12,13,14]$.

High-dose-rate (HDR) brachytherapy and external beam radiotherapy (EBRT) have been described as treat- ment modalities for NMSC $[5,7,15,16]$. HDR brachytherapy is often delivered in the form of a surface mold applied in multiple fractions but can also be delivered through use of a custom mold, Leipzig applicator, Valencia applicator, or a variety of other techniques $[12,13,16]$. EBRT can be delivered in hypofractionated or standard fractionation regimens. Orthovoltage radiotherapy is another mainstay treatment option but requires a dedicated machine for delivery, which limits widespread use and precluded inclusion in this institutional review.

There is little data comparing the three treatment modalities considered herein. This study seeks to examine differences in local control and overall survival among 
patients receiving different modalities of radiotherapy treatment.

\section{Material and methods}

\section{Outcome measures}

An Institutional Review Board-approved, retrospective evaluation was undertaken of patients treated for NMSC from June 2005 to March 2013. Sixty one patients received treatment for 66 primary lesions treated with HDR

Table 1. Patient, treatment, and tumor characteristics

\begin{tabular}{|c|c|c|}
\hline \multirow[t]{2}{*}{ Parameter } & \multicolumn{2}{|c|}{ Overall } \\
\hline & $n$ & $\%$ \\
\hline Total patients & 61 & \\
\hline Age, mean, SD & 83.3 & 11.8 \\
\hline \multicolumn{3}{|l|}{ Gender } \\
\hline Male & 41 & 67.2 \\
\hline Female & 20 & 32.8 \\
\hline \multicolumn{3}{|l|}{ Type of RT } \\
\hline Brachytherapy & 9 & 14.8 \\
\hline Hypofractionation & 30 & 49.2 \\
\hline Standard fractionation & 22 & 36.1 \\
\hline \multicolumn{3}{|l|}{ Local control } \\
\hline Yes & 46 & 75.4 \\
\hline No & 11 & 18.0 \\
\hline Missing data & 4 & 6.6 \\
\hline \multicolumn{3}{|l|}{ Cosmetic outcome } \\
\hline Excellent & 3 & 4.9 \\
\hline Good & 34 & 55.7 \\
\hline Poor & 6 & 9.8 \\
\hline Missing data & 18 & 29.5 \\
\hline \multicolumn{3}{|l|}{ RTOG grade $2+$ toxicity } \\
\hline Yes & 13 & 21.3 \\
\hline No & 47 & 77.1 \\
\hline Missing data & 1 & 1.6 \\
\hline Total tumors & 66 & \\
\hline \multicolumn{3}{|l|}{ Histology } \\
\hline $\mathrm{BCC}$ & 31 & 47.0 \\
\hline SCC & 29 & 43.9 \\
\hline \multicolumn{3}{|l|}{ Other } \\
\hline MCC & 3 & 4.5 \\
\hline Basosquamous CA & 3 & 4.5 \\
\hline
\end{tabular}

brachytherapy $(n=9)$, hypofractionated EBRT $(n=30)$, or standard fractionation EBRT $(n=22)$ as indicated in Table 1 . The primary outcome measure was local control (LC), which, for the purposes of analysis, was assessed at the most remote follow-up appointment. Secondary outcome measures were overall survival (OS), cosmetic outcome, and toxicity.

The treating physician determined cosmetic outcome upon follow-up visit 6 months or greater after completion of radiotherapy. Cosmetic outcome was graded as

\begin{tabular}{lll} 
& \multicolumn{2}{c}{ Overall } \\
\cline { 2 - 3 } & $n$ & $\%$ \\
\hline Histology by treatment modality & & \\
\hline Brachytherapy & & \\
\hline BCC & 6 & 9.1 \\
\hline SCC & 3 & 4.5 \\
\hline Other & 0 & 0
\end{tabular}

Hypofractionation

\begin{tabular}{lcc}
\hline BCC & 16 & 24.2 \\
\hline SCC & 16 & 24.2 \\
\hline Other & 1 & 1.5
\end{tabular}

Standard fractionation

\begin{tabular}{lcc}
\hline BCC & 9 & 13.6 \\
\hline SCC & 10 & 15.2 \\
\hline Other & 5 & 7.6
\end{tabular}

Tumor location

$\begin{array}{lll}\text { Nose } & 15 & 22.7\end{array}$

\begin{tabular}{lll}
\hline Scalp & 13 & 19.7
\end{tabular}

\begin{tabular}{lll}
\hline Cheek & 10 & 15.2
\end{tabular}

Lip $\quad 4.1$

Eyelid 4 ( 6.1

Ear $\quad 3 \quad 4.6$

\begin{tabular}{lll}
\hline Neck & 3 & 4.6
\end{tabular}

\begin{tabular}{lll}
\hline Back & 3 & 4.6
\end{tabular}

\begin{tabular}{lll}
\hline Shoulder & 2 & 3.0
\end{tabular}

\begin{tabular}{lll}
\hline Arm & 1 & 1.5 \\
\hline
\end{tabular}

\begin{tabular}{lll}
\hline Leg/Ankle/Foot & 8 & 12.1
\end{tabular}

Tumor size, median, range $(\mathrm{cm})$

\begin{tabular}{lcc}
\hline Brachytherapy & 2.3 & $0.5-6$ \\
\hline Hypofractionation & 1.8 & $0.3-6$ \\
\hline Standard fractionation & 2 & $0.8-8$
\end{tabular}

SD - standard deviation, SCC - squamous cell carcinoma, BCC - basal cell carcinoma, RTOG - Radiation Therapy Oncology Group 
excellent if there was no telangiectasia or fibrosis and no or slight pigment change, good if there was mild telangiectasia or pigment change or mild-to-moderate fibrosis, and poor if there was severe fibrosis or skin contracture. Skin toxicity was measured using Radiation Therapy Oncology Group (RTOG) acute radiation morbidity scoring criteria. Toxicities of grade 2 and greater were considered clinically meaningful for this analysis. Patient demographic and tumor and dose characteristic data was also collected including patient age and gender, tumor histology, tumor size, total dose, number of fractions, and dose per fraction. Median follow-up was 30 months.

\section{Treatment}

HDR brachytherapy was delivered as a surface mold using an iridium-192 isotope as previously described [10]. Briefly, a surface mold of the tumor is constructed of pliable material, such as silicone or polymethyl-methacrylate (Figure 1). Dose distribution calculations were performed to determine optimal placement of radioactive sources on the surface mold to obtain uniform dose coverage throughout the tumor volume. Treatment was delivered in daily fractions.

EBRT treatment was performed with a linear accelerator manufactured by Varian (Palo Alto, USA). Electrons were delivered as 6-12 MeV beams and were shape using custom Wood's metal blocks to improve conformation. Standard fractionation was defined as fractional doses of 1.8-2.0 Gy. Hypofractionation was defined as fractional doses of 2.5 Gy or greater.

Choice of radiation treatment modality was largely based on two factors. The first was which modality was likely to obtain the most favorable dosimetric distribution; uneven surfaces such as the nasal ala may compromise electron dose distribution. Such lesions favor treatment with brachytherapy, which can provide more uniform dose to uneven locations. The second consideration was feasibility of treatment for the patient. Patients who were unable to come for standard fractionation therapy were likely to be recommended to undergo brachytherapy or hypofractionated EBRT. Dose characteristics for all treatment modalities are in Table 2.

\section{Statistical analysis}

Univariate analysis was performed to determine association of treatment modality and tumor histology with LC using Pearson Chi-square test. Univariate analysis was performed to determine association of treatment modality with cosmetic outcome and toxicity using Pearson Chi-square test. Univariate analysis was performed to

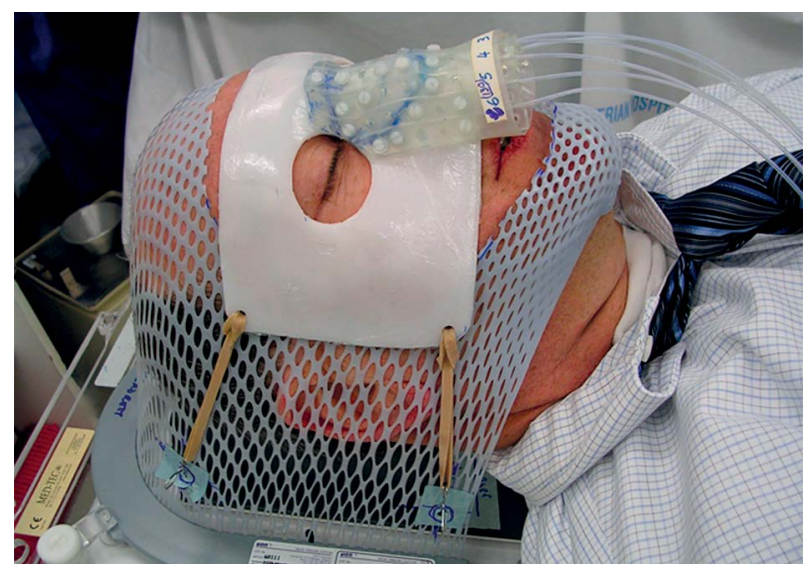

Fig. 1. A representation of a nasal HDR applicator for treatment of skin cancer of the nose

determine association of age with cosmetic outcome and toxicity using ANOVA and Student $t$-test, respectively. Multivariate analysis was not undertaken due to the relatively small number of outcome events in the study.

OS was assessed using Kaplan-Meier analysis and comparison was made between treatment modalities with Log-Rank testing.

\section{Results}

\section{Local control}

Overall rate of LC was $81 \%$ among patients with LC data. Treatment with brachytherapy achieved $100 \%$ local control but no significant association was found between treatment modality and local control (Table 3). No significant association was found for LC between BCC and SCC histologies when compared directly; however, LC was significantly reduced among tumors having Merkel cell and basosquamous histologies $(p=0.03$; Table 3$)$.

\section{Overall survival}

OS data was available for 40 patients and median follow-up for patients with OS data was 30 months. 2-year actuarial OS was $89 \%$ and 3-year actuarial OS was $79 \%$. OS was not significantly associated with treatment modality either when comparing all modalities ( $p=0.66$; Figure 2) or when performing pair-wise comparisons.

\section{Cosmetic outcome and RTOG grade $\geq 2$ toxicity}

Cosmetic outcome was excellent or good for $86 \%$ of patients. Five out of six patients noted to have a poor cosmetic outcome were treated with standard fractionation

Table 2. Treatment parameters for brachytherapy, hypofractionated EBRT, and standard fractionation EBRT

\begin{tabular}{lccc} 
Treatment modality & Median dose (Gy) & Dose range (Gy) & Treatment duration (number fx) \\
\hline Brachytherapy & 40 & $30-40$ & $8-10$ \\
\hline Hypofractionated EBRT & 45 & $13.1-52.75$ & $5-21$ \\
\hline Standard fractionation EBRT & 59.4 & $9-70$ & $5-35$
\end{tabular}

EBRT - external beam radiotherapy; Gy - gray; $f x$ - fractions 
Table 3. Univariate analysis for local control

\begin{tabular}{|c|c|c|c|c|c|}
\hline & \multicolumn{4}{|c|}{ Local control } & \multirow[t]{3}{*}{$p$ value } \\
\hline & \multicolumn{2}{|c|}{ Yes } & \multicolumn{2}{|c|}{ No } & \\
\hline & $n$ & $\%$ & $n$ & $\%$ & \\
\hline Total & 46 & & 11 & & \\
\hline Type of RT & & & & & 0.43 \\
\hline Brachytherapy & 8 & 100.0 & 0 & 0.0 & \\
\hline Hypofractionation & 22 & 75.9 & 7 & 24.1 & \\
\hline Standard fractionation & 16 & 80.0 & 4 & 20.0 & \\
\hline Histology & & & & & 0.03 \\
\hline SCC & 22 & 84.6 & 4 & 15.4 & \\
\hline $\mathrm{BCC}$ & 22 & 88.0 & 3 & 12.0 & \\
\hline \multicolumn{6}{|l|}{ Other } \\
\hline$\overline{M C C}$ & 1 & 33.3 & 2 & 66.7 & \\
\hline Basosquamous CA & 1 & 33.3 & 2 & 66.7 & \\
\hline
\end{tabular}

SCC - squamous cell carcinoma; BCC - basal cell carcinoma

EBRT, however no significant difference in cosmetic outcome was found when comparing all treatment modalities (Table 4). More favorable cosmetic outcomes tended to be observed in older patients but cosmetic outcome was not significantly associated with patient age.

RTOG grade $\geq 2$ toxicity was observed in $22 \%$ of patients. No patients had grade 4 or greater toxic events. Grade $\geq 2$ toxicity rate was observed to be greatest among patients treated with standard fractionation EBRT (38\%) and least among patients treated with hypofractionation EBRT $(10 \%)$, however no significant difference in grade $\geq 2$ toxicity was found when comparing all treatment mo-

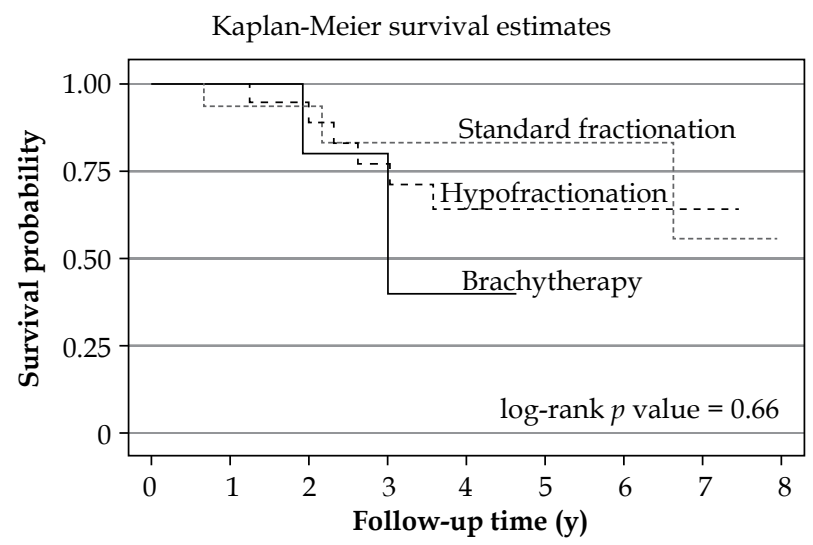

Number at risk

Brachytherapy

\begin{tabular}{|c|c|c|c|c|c|c|c|}
\hline 8 & 5 & 4 & 2 & 1 & 0 & 0 & 0 \\
\hline \multicolumn{8}{|c|}{ Hypofractionation } \\
\hline 22 & 18 & 17 & 13 & 9 & 5 & 3 & 1 \\
\hline \multicolumn{8}{|c|}{ Standard fractionation } \\
\hline 19 & 13 & 11 & 6 & 5 & 3 & 3 & 2 \\
\hline
\end{tabular}

Fig. 2. Kaplan-Meier survival by treatment modality dalities (Table 5). Patient age was not significantly associated with grade $\geq 2$ toxicity.

Grade 2 toxicity was largely related to skin desquamation. Mucositis was observed in some patients with facial lesions, and some patients with periorbital lesions experienced conjunctivitis. Most patients experienced resolution of symptoms following completion of RT. Two patients experienced grade 3 toxicity. One patient had facial cellulitis treated with antibiotics, and the other patient had mucositis, difficulty swallowing, and extreme fatigue, which resolved upon cessation of EBRT. Both patients with grade 3 toxicity were treated with standard fractionation EBRT.

\section{Discussion}

The rate of local control obtained in treatment of BCC and SCC with radiotherapy has been observed to be in the range of $92-97 \%$ in previous analyses of EBRT [4,7] and HDR brachytherapy [17]. This analysis found somewhat reduced LC at a rate of $81 \%$. One possible explanation is that several of the tumors treated in this review were quite large, ranging in size up to $8 \mathrm{~cm}$ in greatest dimension. Tumors of such a large size may not be ideally suited for treatment with radiotherapy. There may be other patient selection factors that were not assessed that additionally contributed to the reduced LC. Of note, brachytherapy achieved a LC of $100 \%$ in this study, which is consistent with very high levels of LC obtained in previous studies of HDR brachytherapy [17]. Although the rate of LC observed among patients treated with brachytherapy was high, the overall LC rate for the study was lower since brachytherapy was utilized in only $15 \%$ of the patient population. No statistical difference in LC between treatment modalities was observed in this study. 
Table 4. Univariate analysis for cosmetic outcome

\begin{tabular}{|c|c|c|c|c|c|c|c|}
\hline & \multicolumn{6}{|c|}{ Cosmetic outcome } & \multirow[t]{3}{*}{$p$ value } \\
\hline & \multicolumn{2}{|c|}{ Excellent } & \multicolumn{2}{|c|}{ Good } & \multicolumn{2}{|c|}{ Poor } & \\
\hline & $n$ & $\%$ & $n$ & $\%$ & $n$ & $\%$ & \\
\hline & 3 & & 34 & & 6 & & \\
\hline Type of RT & & & & & & & 0.16 \\
\hline Brachytherapy & 1 & 20.0 & 4 & 80.0 & 0 & 0.0 & \\
\hline Hypofractionation & 1 & 5.0 & 18 & 90.0 & 1 & 5.0 & \\
\hline Standard fractionation & 1 & 5.6 & 12 & 66.7 & 5 & 27.8 & \\
\hline Age, mean, SD & 90.00 & 5.29 & 84.44 & 10.19 & 77.33 & 6.31 & 0.14 \\
\hline
\end{tabular}

SD-standard deviation

Table 5. Univariate analysis for toxicity

\begin{tabular}{|c|c|c|c|c|c|}
\hline & \multicolumn{4}{|c|}{ RTOG grade $2+$ toxicity } & \multirow[t]{3}{*}{$p$ value } \\
\hline & \multicolumn{2}{|c|}{ Yes } & \multicolumn{2}{|c|}{ No } & \\
\hline & $n$ & $\%$ & $n$ & $\%$ & \\
\hline & 13 & & 47 & & \\
\hline Type of RT & & & & & 0.06 \\
\hline Brachytherapy & 2 & 22.2 & 7 & 77.8 & \\
\hline Hypofractionation & 3 & 10.0 & 27 & 90.0 & \\
\hline Standard fractionation & 8 & 38.1 & 13 & 61.9 & \\
\hline Age, mean, SD & 79.54 & 9.88 & 84.36 & 12.31 & 0.20 \\
\hline
\end{tabular}

SD - standard deviation; RTOG - Radiation Therapy Oncology Group

To our knowledge, this is the first analysis to compare brachytherapy, hypofractionated EBRT, and standard fractionation EBRT. A review of the literature identified one previous analysis that assessed for a difference in LC between EBRT and orthovoltage RT. Four hundred sixty eight patients with 531 lesions of BCC or SCC were analyzed. After median follow-up of 5.8 years, overall LC rate was $89 \%$ [5]. Greater dose per fraction was associated with greater LC but no difference was detected in LC between treatment modalities.

SCC is considered to represent a more aggressive histology than BCC, and one analysis observed reduced LC rate of $80 \%$ among SCC treated with EBRT [5]. The present study observed no difference in LC between BCC and SCC, which is consistent with the prevailing literature. Among NMSC histologies, MCC is considered aggressive [18]. The finding in this study of greatly reduced LC among patient with MCC is consistent with the known biology, however the LC rate is not necessarily representative given the low number of tumors with Merkel cell histology. Basosquamous histology is considered to be intermediate between BCC and SCC and should display concomitantly intermediate aggression [19]. It is thus not expected that basosquamous tumors in this analysis display a low rate of LC. This finding is likely due to the small number of tumors with basosquamous histology.
Cosmetic outcome in this study was favorable with $86 \%$ of patients achieving excellent or good outcome. This is consistent with previous reports, which have found excellent or good outcome in $92 \%$ [5] and $83-87 \%$ [7] of patients. Although no significant difference was observed in cosmetic outcome between treatment modalities, the patients treated with standard fractionation accounted for five of the six poor cosmetic outcomes observed, indicating that this treatment modality may tend to yield poorer cosmetic outcomes than the others. Paradoxically, cosmetic outcome tended to be better in older patients, however this finding was not significant and is likely spurious. Treatment with brachytherapy tended to yield quite good cosmetic outcomes. Though the finding is not significant, it may be speculated that shallower treatment and smaller margins perhaps contributed to better quality cosmesis.

Treatment was overall well tolerated with $22 \%$ of patients experiencing RTOG grade $\geq 2$ toxicity. Patients treated with hypofractionated EBRT fared the best with only $10 \%$ grade $\geq 2$ toxicity, while patients treated with standard fractionation experienced nearly $40 \%$ grade $\geq 2$ toxicity. Additionally, the only two patients who experienced grade 3 toxicity were treated with standard fractionation EBRT. The finding of higher grade toxicity and a greater portion of patients experiencing toxicity among 
standard fractionation therapy is counter to expectations. There was no statistical significance to the finding and it is not likely to be meaningful. Radiobiologically, one would be led to believe that a hypofractionated schedule should result in greater toxicity in light of expected late effects of radiation. It is not clear why the opposite finding was observed; perhaps it is the result of a small sample size.

Data for overall survival in NMSC treated with RT is relatively sparse. This is likely related to the generally favorable prognosis for NMSC and the long follow-up required to observe adverse events. One report was found of 333 patients treated for 434 lesions of BCC or SCC with two different hypofractionated schedules of EBRT. One group of patients was treated with $54 \mathrm{~Gy}$ in 18 fractions of $3 \mathrm{~Gy}$ while the other group was treated with $44 \mathrm{~Gy}$ in 10 fractions of $4.4 \mathrm{~Gy}$. After median follow-up of 42.8 months, 3-year OS of 75\% was observed [7]. No significant difference in LC between study groups was detected; difference in OS between study groups was not reported. The 2-year OS of $89 \%$ and 3-year OS of $79 \%$ observed in this study is moderately more favorable but essentially consistent with the previously reported OS.

This study observed no difference in OS when comparing all three treatment modalities or when conducting pair-wise comparison of treatment modalities. Although the Kaplan-Meier curves in Figure 2 diverge somewhat after 3 years of follow-up, this is likely due to the low number of remaining patients at risk, making the plot more unpredictable. There were relatively few total events for OS analysis, which likely made detection of any existing difference between modalities more difficult.

\section{Conclusions}

This study found no difference in local control or overall survival between patients treated for NMSC with brachytherapy, hypofractionated EBRT, and standard fractionation EBRT. Standard fractionation EBRT was found to have a non-significantly increased rate of RTOG grade $\geq 2$ toxicity as compared to other treatment modalities, and the greatest proportion of poor cosmetic outcome also occurred in patients treated with standard fractionation EBRT. However, these findings are counter to expectations, and given that, they lack statistical significance, it is unlikely that such a result is meaningful. More investigation should be pursued to parse out treatment response and toxicity.

\section{Disclosure}

Authors report no conflict of interest.

\section{References}

1. Rogers HW, Weinstock MA, Harris AR et al. Incidence estimate of nonmelanoma skin cancer in the United States, 2006. Arch Dermatol 2010; 146: 283-287.

2. Haseltine JM, Wernicke AG, Formenti SC et al. Treatment of Non-Melanomatous Skin Cancer with Radiotherapy. Curr Dermatol Rep 2015; 4: 187-194.

3. Bath-Hextall FJ, Perkins W, Bong J et al. Interventions for basal cell carcinoma of the skin. Cochrane database Syst Rev 2007; (1): CD003412.
4. Schulte KW, Lippold A, Auras C et al. Soft x-ray therapy for cutaneous basal cell and squamous cell carcinomas. J Am Acad Dermatol 2005; 53: 993-1001.

5. Locke J, Karimpour S, Young G et al. Radiotherapy for epithelial skin cancer. Int J Radiat Oncol Biol Phys 2001; 51: 748-755.

6. Caccialanza M, Piccinno R, Moretti D et al. Radiotherapy of carcinomas of the skin overlying the cartilage of the nose: results in 405 lesions. Eur J Dermatol 2003; 13: 462-465.

7. Van Hezewijk M, Creutzberg CL, Putter H et al. Efficacy of a hypofractionated schedule in electron beam radiotherapy for epithelial skin cancer: Analysis of 434 cases. Radiother Oncol 2010; 95: 245-249.

8. Thanh Pham T, Cross S, Gebski V et al. Squamous cell carcinoma of the lip in Australian patients: definitive radiotherapy is an efficacious option to surgery in select patients. Dermatol Surg 2015; 41: 219-225.

9. Zagrodnik B, Kempf W, Seifert B et al. Superficial radiotherapy for patients with basal cell carcinoma: recurrence rates, histologic subtypes, and expression of p53 and Bcl-2. Cancer 2003; 98: 2708-2714.

10. Nguyen NP, Ries T, Vock J et al. Effectiveness of radiotherapy for elderly patients with non-melanoma skin cancer of the head. Geriatr Gerontol Int 2015; 15: 601-605.

11. Frakulli R, Galuppi A, Cammelli S et al. Brachytherapy in non melanoma skin cancer of the eye: a systematic review. J Contemp Brachytherapy 2015; 7: 497-502.

12. Delishaj D, Laliscia C, Manfredi B et al. Non-melanoma skin cancer treated with high-dose-rate brachytherapy and Valencia applicator in elderly patients: a retrospective case series. J Contemp Brachytherapy 2015; 7: 437-444.

13. Tormo A, Celada F, Rodriguez $S$ et al. Non-melanoma skin cancer treated with HDR Valencia applicator: clinical outcomes. J Contemp Brachytherapy 2014; 6: 167-172.

14. Goyal U, Kim Y, Tiwari HA et al. A pilot study of ultrasound-guided electronic brachytherapy for skin cancer. J Contemp Brachytherapy 2015; 7: 374-380.

15. Alam M, Nanda S, Mittal BB et al. The use of brachytherapy in the treatment of nonmelanoma skin cancer: a review. J Am Acad Dermatol 2011; 65: 377-388.

16. Ouhib Z, Kasper M, Perez Calatayud J et al. Aspects of dosimetry and clinical practice of skin brachytherapy: The American Brachytherapy Society working group report. Brachytherapy 2015; 14: 840-858.

17. Guix B, Finestres F, Tello J et al. Treatment of skin carcinomas of the face by high-dose-rate brachytherapy and custom-made surface molds. Int J Radiat Oncol Biol Phys 2000; 47: 95-102.

18. Becker JC, Schrama D, Houben R. Merkel cell carcinoma. Cell Mol Life Sci 2009; 66: 1-8.

19. Garcia C, Poletti E, Crowson AN. Basosquamous carcinoma. J Am Acad Dermatol 2009; 60: 137-143. 